bioRxiv preprint doi: https://doi.org/10.1101/634113; this version posted May 10, 2019. The copyright holder for this preprint (which was not

certified by peer review) is the author/funder. All rights reserved. No reuse allowed without permission.

\title{
Cross-talk between SIM2s and NFkB regulates cyclooxygenase 2 expression in breast cancer
}

Garhett Wyatt ${ }^{1}$, Chloe Young ${ }^{3}$, Lyndsey Crump ${ }^{3}$, Veronica Wessells ${ }^{3}$, Tanya Gustafson ${ }^{1}$, Yang-Yi Fan ${ }^{2}$, Robert Chapkin $^{2}$, Weston Porter ${ }^{1^{*}}$ and Traci R Lyons ${ }^{3^{*}}$

${ }^{1}$ Department of Veterinary Integrative Biosciences and ${ }^{2}$ Department of Nutrition, Texas A\&M University, College Station, TX, USA, ${ }^{3}$ University of Colorado Anschutz Medical Campus, School of Medicine Department of Medicine, Division of Medical Oncology, Aurora, CO, USA, and ${ }^{4}$ The University of Colorado Cancer Center Young Women's Breast Cancer Translational Program, Aurora, CO, USA.

*denotes co-corresponding authors

Address correspondence to:

Weston Porter, wporter@cvm.tamu.edu

Traci R Lyons, traci.Iyons@ucdenver.edu

\begin{abstract}
Background: Breast cancer is a leading cause of cancer-related death for women in the United States. Thus, there a need to investigate novel prognostic markers and therapeutic strategies. Inflammation raises challenges to both treating and preventing the spread of breast cancer. Specifically, the nuclear factor kappa $b$ (NFkB) pathway contributes to cancer progression by stimulating proliferation and preventing apoptosis. One target gene of this pathway is PTGS2, the gene that encodes for cyclooxygenase 2 (COX-2), which is upregulated in $40 \%$ of human breast carcinomas. COX-2 is an enzyme involved in inflammation. Here we investigate the effect of Singleminded 2s, a transcriptional tumor suppressor that is implicated in inhibition of tumor growth and metastasis, in regulating NFkB and COX-2. Methods: We utilized in vitro reporter assays, immunoblot analyses, qPCR and immunohistochemical analysis to dissect the relationship between NFkB, SIM2s, and COX-2. Furthermore, we utilized COX-2 targeting strategies to determine tumor suppressive activities. Results: Our results reveal that SIM2s attenuates the activation of a NFKB via luciferase reporter assays. Furthermore, immunostaining of lysates from breast cancer cells over expressing SIM2s showed reduction in various NFKB signaling proteins, whereas knockdown of SIM2 revealed increases in the same NFKB signaling proteins. Additionally, by increasing NFKB translocation to the nucleus in DCIS.COM cells, we show that NFKB signaling can act in a reciprocal manner to decrease expression of SIM2s. Likewise, suppressing NFkB translocation in DCIS.COM cells increases SIM2s expression. We also found that NFKB/p65 represses SIM2 in via dose-dependent manner and when NFKB is suppressed the effect on the SIM2 is negated. Additionally, our CHIP analysis confirms that NFKB/p65 binds directly to SIM2 promoter site and that the NFkB sites in the SIM2 promoter are required for NFkB-mediated suppression of SIM2s. Finally, over expression of SIM2s decreases PTGS2 in vitro and COX-2 staining in vivo while decreasing PTGS2 and/or Cox-2 activity results in re-expression of SIM2. Our findings identify a novel role for SIM2s in NFKB signaling and COX-2 expression. Conclusions: These findings provide evidence for a mechanism where SIM2s may represses COX-2 expression to provide an overall better prognosis for breast cancer patients.
\end{abstract}

\section{Keywords}

SIM2, COX-2, NFkB, Inflammation, Breast Cancer, Prostaglandin $\mathrm{E}_{2}$ 


\section{Background:}

Despite improvement in early detection and treatment, breast cancer remains the second leading cause of cancer-related death for women in the United States [1]. Breast ductal carcinoma in situ, referred to as DCIS, consists of a heterogeneous group of diseases characterized by a neoplastic mammary lesion that is confined to the ductal system of the breast [2]. DCIS progresses to invasive ductal carcinoma (IDC) through events such as epithelial mesenchymal transition (EMT), basement membrane degradation, controlled inflammatory signaling and other pathways associated with a wound-healing milieu [3-5]. It is estimated that $\sim 20 \%$ of mammography detected breast cancers are DCIS[6] and ACS Facts and Figures indicates $\sim 65,000$ cases of DCIS are diagnosed per year[7]. Provided that DCIS is removed surgically, as is standard of care, a woman diagnosed with DCIS without recurrence is more likely to die of other causes than of breast cancer[8]. However, it is estimated that $\sim 15-20 \%$, or $\sim 10-13,000$, DCIS patients recur with invasive disease within a decade[9, 10]. Recently identified risk factors for DCIS recurrence include age $<40$ at diagnosis, African American ethnicity, hormone receptor negativity, and HER2 positivity[8]. However, these high-risk groups only account for $20 \%$ of the DCIS patient population[10]. Therefore, identifying additional risk factors for, or markers that will predict, DCIS aggressiveness are extremely important goals for preventing invasive cancer in DCIS patients.

There is increasing evidence that inflammation plays a key role in breast cancer progression [11]. One such specific inflammatory pathway is nuclear factor kappa $b(N F K B)$. The NFKB signaling pathway includes five members: NFKB1 (p105/p50), NFKB2 (p100/p52), RelA (p65), RelB and c-Rel. Dimers of the aforementioned proteins are held in the cytoplasm by inhibitor kappaB (lkB) proteins, primarily the lkBa protein. The mechanism of action for NFKB includes phosphorylation of IkBa by inhibitor of kappaB kinase (most commonly $\mathrm{IKK} \alpha$ and IKK $\beta$ ), which leads to degradation of IkBa. Upon degradation of the IkBa, NFKB heterodimers, specifically the canonical heterodimer p50/p65, translocate to the nucleus and bind to promoters of certain target genes, leading to activation of transcription[12,13]. Two NFKB consensus sites are located in the promoter region of the human PTGS2 gene, which encodes for pro-inflammatory enzyme COX-2[14]. These NFKB sites are conserved on the 5'-promoter regions in both mouse and human, however the 3' promoter is only found in human[15]. These NFKB consensus sites not only contribute to cancer progression by stimulating and preventing apoptosis, but also the activation of cyclooxygenase 2 (COX-2) mediated signaling [16]. COX2 is the inducible form of cyclooxygenase, which is the key enzyme involved in the biosynthesis of the proinflammatory agent prostaglandin[17-22]. COX-2 has been implicated in DCIS progression through promotion of mammary tumorigenesis via increases in proliferation, migration, invasion and metastatic spread in preclinical models[23-25]. Additionally, expression of COX-2 is frequently observed in patients with invasive disease and is associated with DCIS recurrence. Furthermore, therapeutic benefit of inhibiting COX-2 has been observed in colon, esophagus, lung, bladder, breast and prostate cancers[19, 20, 26-36]. Thus, it is logical to expect that inhibition of COX-2 in breast cancer patients could enhance overall prognosis.

We have shown that Singleminded-2s (SIM2s; expressed from SIM2), a member of the bHLH/PAS family of transcription factors, is a tumor suppressor that is expressed in breast epithelial cells and down-regulated in the transition from DCIS to IDC [37-40]. Specifically, using the DCIS.com progression model we demonstrated that re-expression of SIM2s inhibits growth, invasive phenotypes, and progression to metastasis. Furthermore, expression of SIM2s promotes a more luminal-like phenotype in breast cancer cells whereas down-regulation of SIM2s leads to an increase in invasive potential [40]. Consistent with the role for SIM2s in cancer progression, we have also shown that the NFKB signaling pathway is downregulated by SIM2s over expression during mammary development, specifically during postpartum mammary involution[41]. In this study, we expand on these observations and demonstrate a relationship between SIM2s, the NFKB signaling pathway, and COX-2. We suggest that re-expression of SIM2s could be mediated by inhibition of COX-2 signaling, which may serve to reduce breast cancer progression.

\section{Methods:}

\section{Cell Culture}

MCF7 and Sum159 cells were purchased from American Type Culture Collection (ATCC) and were maintained in accordance to their guidelines. MCF10-DCIS.com cells were generously donated by Dr. Dan Medina (Baylor College of Medicine, Houston, TX, USA). Cells were plated in 6 well plates for RNA isolation experiments according the guidelines from ThermoFisher Scientific. Celecoxib experiment were performed as follows; cells 
were first plated in $10 \mathrm{uM}$ celecoxib for 24 hours, then media was changed and treatment was performed at 20 uM celecoxib for 24 hours and then harvested for analysis. DHA experiments on cell lines were performed as follows; cells were dosed with 50uM DHA for 24 hours and then harvested for analysis.

\section{Generation of cell lines}

Point mutations in the SIM2 gene were generated via long cDNA synthesis (Invitrogen). Plasmids were amplified using Subcloning Efficiency DH5a competent cells (Life Technologies). Plasmid DNA was isolated using the HiPure Plasmid Maxiprep kit (Life Technologies) or the ZymoPURE Plasmid DNA Isolation Kit (Zymo Research). Viral transduction was then performed as previously described [39]. Puromycin selection (2ug/mL) was started the following day and maintained for a week.

\section{Animal Models}

200,000 MCF10DCIS cells stably expressing anti COX-2 shRNAs (a generous gift from Kornelia Polyak and Andriy Marusyk) were orthotopically injected and tumors harvested as previously described [23, 24].

\section{RNA isolation and real-time PCR}

As previously described [42]. Primers can be found in Supplementary Table 2.

\section{Immunoblotting}

Cells were washed with cold PBS and lysed in high-salt lysis buffer (50mM HEPES, 500mM NaCl, $1.5 \mathrm{mM}$ $\mathrm{MgCl} 2,1 \mathrm{mM}$ thylenediaminetetraacetic acid (EDTA), 10\% glycerol, 1\% Triton X-100, pH 7.5) supplemented with $1 \mathrm{mM} \mathrm{Na}_{3} \mathrm{VO}_{4}$ (Sigma) and $1 \mathrm{mM}$ complete ULTRA tablets mini EDTA-free Easy pack (Roche). Protein concentration was determined using the DC Protein Assay (Bio-Rad) with bovine serum albumin as a standard. Immunoblotting and zymography were then conducted as previously described[39]. Antibodies can be found in supplementary Table 1. Blots were imaged on a ChemiDoc MP (Bio-Rad) after incubating in ProSignal Pico ECL Spray (Genesee Scientific) for 3 minutes. Quantification performed using ImageJ.

\section{Immunohistochemistry}

IHC for COX-2 was performed as previously described [23]. Analysis for positive nuclei was performed previously described[25]. Antibodies can be found in supplementary Table 1.

\section{Transient transfection}

293T cells were used for all transfections for luciferase activity. One hundred $\mathrm{ng}(0.1 \mu \mathrm{g})$ of plasmid containing transcription factor was mixed with up to $1 \mu \mathrm{g}$ (amount varies per construct) of plasmid containing promoter construct. Three $\mu$ l of Genejuice (Novagen) was used per microgram of DNA. DNA and Genejuice were mixed in Opti-MEM media (Invitrogen). Protein was harvested 2 days after transfection, using Reporter Lysis Buffer (Promega). Luciferase activity and total protein were measured as described previously [38].

Luciferase activities were normalized to total protein values and are represented as the means \pm SE for three wells per condition.

\section{Chromatin Immunoprecipitation}

For ChIP assays, $2 \mu \mathrm{g}$ of plasmid containing repressor and $2 \mu \mathrm{g}$ of plasmid containing the SIM2 promoter construct were transfected into $293 \mathrm{~T}$ cells on a $10 \mathrm{~cm}$ plate. Chromatin was harvested 2 days after transfection.

\section{Statistical Analysis}

To address scientific rigor, all experiments in cell lines and xenografts were conducted in biological triplicates at a minimum, technical duplicates, and repeated three times. Normal distribution was confirmed before conducting unpaired $t$ test. Significance was considered at $p<0.05$ unless otherwise noted.

\section{Results:}

SIM2s downregulates NFKB signaling. To test the hypothesis that SIM2 directly affects NFKB/p65 mediated transcription, we co-transfected a reporter plasmid encoding a NFkB binding site upstream of the luciferase gene (5X NFKB-luc) with the p65 subunit along with SIM2s in 293T cells and measured relative light units as a readout for NFKB activity. As expected, p65 strongly activated the reporter construct; however, this response 
was blocked by co-transfection of SIM2s (Fig 1A). To determine the mechanism of this inhibition, the transfection was repeated with a SIM2s expression construct missing the Pro/Ala transcriptional repression domain (SIM2s $\Delta R$ ). This construct also significantly attenuated the activation of the $5 \mathrm{X}$ NFKB-luc construct by $\mathrm{NFkB} / \mathrm{p} 65$, demonstrating that the repression domain of SIM2s is not required for inhibition of NFKB signaling (Fig 1B). Alternatively, to determine whether SIM2 modulates expression levels of mediators of the NFKB pathway in our breast cancer cell lines to downregulate signaling, we performed western blot analysis and found that IKKa, IKKb, phosphorylated-p65 and p65 protein levels were all decreased in SIM2s overexpressing SUM159-cells (Figure 1C). Similarly, we found that NFKB pathway protein levels were increased in SIM2s knockdown MCF7 cells (Figure 1C). These results suggest that SIM2s may affect NFKB mediated transcription via modulation of expression of key mediators of NFKB signaling.

NFKB signaling downregulates SIM2s expression. We also observed that stable over expression of an inhibitor of the inhibitor kappa kinase beta (IKK $\beta$ ), which normally retains NFKB in the cytoplasm, significantly decreases SIM2s gene expression in the DCIS.com cells (Figure 2A). Likewise, when the NFKB pathway is suppressed via stable transduction of the inhibitor of kappaB alpha super repressor (IKB-SR), which maintains the NFKB heterodimer (p50/p65) in the cytosol, SIM2s expression was increased (Figure 2B). To test the hypothesis that NFKB downregulates SIM2 expression, we cloned a $2 \mathrm{~kb}$ portion of the SIM2 promoter upstream of the luciferase gene and co-transfected with increasing amounts of p65 in HEK293T cells. We observed dose-dependent repression of SIM2s promoter activity (Figure 2C). Importantly, co-transfection with IKB-Super Repressor (IKB-SR), as well as IKB-SR with NFkB p65, successfully reversed the downregulation of SIM2s promoter activity (Figure 2D) suggesting that this was not a dominant negative effect. Subsequently, analysis of the SIM2 promoter identified two consensus NFkB binding sites near the transcriptional start site for SIM2. Thus, we utilized chromatin immunoprecipitation (ChIP) analysis to show that p65 directly binds to the SIM2 promoter around the transcriptional start site (Figure 2E). Then, to determine whether binding of p65 to the NFkB binding sites is necessary for downregulation of SIM2s expression, we mutated the two NFKB sites in the SIM2 promoter reporter construct and performed additional co-transfection experiments with p65. Interestingly, the NFKB double mutant promoter failed reduce SIM2 promoter activity when compared to the wild-type promoter (Figure 2F), implicating a direct interaction of NFKB/p65 to decrease SIM2 transcription. These results suggest that NFKB mediated transcriptional repression of SIM2s may allow for activation of NFKB mediated transcription of pro-inflammatory target genes such as PTGS2.

SIM2s expression downregulates COX2. To explore the relationship between SIM2s and PTGS2 expression in breast cancer we analyzed three different breast cancer cell lines including MCF7, MCF10ADCIS.COM (DCIS.COM), and SUM159 cells. The non-invasive MCF7 cell line and the highly invasive triple-negative SUM159 cell line were utilized to examine the differential expression of SIM2, and subsequent regulation PTGS2, as it relates to invasion. DCIS.COM cells were also used for their unique ability to mimic basal-like DCIS in vivo and their ability to progress to invasive disease upon acquisition of COX-2 protein expression[23, 43]. We have previously shown that the invasive competent DCIS.COM cells have more SIM2 expression when compared with the non-invasive MCF7 [38, 39]. Confirming and extending this observation, qPCR analysis reveals lowest PTGS2 expression in MCF7 cells, which was increased 130-fold in DCIS.COM cells and highest in in the SUM159 cells (SFigure1A). To determine whether reduction of SIM2s in the non-invasive cells could increase expression of PTGS2, we analyzed control and shRNA-SIM2s DCIS.COM and MCF7 cells by qPCR. Our results revealed that downregulation of SIM2s led to a significant increase in PTGS2 gene expression in both cell lines (Fig 3A, Fig 3B). Moreover, we found that over expression of SIM2s in highly invasive SUM159 cells significantly inhibited PTGS2 expression (Fig 3C). In our previous studies, we showed that over expression of SIM2s in DCIS.COM cells blocked invasion in vivo, whereas loss of SIM2s or over expression of the protein product of PTGS2, COX-2, resulted in increased invasion and metastasis[23, 42]. To determine the relationship between SIM2s and COX-2 protein expression, we performed immunohistochemical (IHC) analysis for COX-2 in tumors derived from control and SIM2s DCIS.COM xenografts to show that COX-2 levels were decreased with over expression of SIM2s in vivo (Fig 3D). Taken together these results suggest that SIM2s may repress invasion in the DCIS.COM model by promoting downregulation of COX-2.

COX-2 downregulation restores SIM2s. Since the invasive potential in DCIS.COM positively correlates with, and depends upon expression and activity of, COX-2 [23] we proposed to test the hypothesis that the loss of invasive phenotype observed with blocking of COX-2 expression and/or activity was due, in part, to re- 
expression of SIM2s. Thus, we measured SIM2 protein levels in cells with confirmed stable knockdown of PTGS2/COX-2 via immunoblot. Indeed, DCIS.COM shPTGS2 and control cells exhibited and inverse relationship between SIM2s and COX-2(Figure 4A). Extending these observations IHC analysis of tumors generated from control and shPTGS2 DCIS.COM cells, which are less invasive[23], revealed an increase in positive nuclear staining for SIM2 with PTGS2 knockdown (Figure 4B\&C). To determine whether COX-2 activity drives the inverse relationship between SIM2 and COX-2 and cell invasion, we treated the highly invasive SUM159 cells with a dose of the selective COX-2 inhibitor, celecoxib, that had previously been shown to decrease invasion of COX-2 expressing cells[23]. We observed a significant increase in SIM2 expression (Figure 4D). Additionally, we show that Docosahexaenoic (DHA), a n-3 polyunsaturated fatty acids (PUFA) that can result in a shift to a more anti-inflammatory gene expression profile[44], and can reduce COX-2 expression[45-48], significantly increases SIM2s expression (Figure 4E). Thus, our driving hypothesis is that reduction of inflammatory pathways via inhibition of activity and/or decreased COX-2 expression results in reexpression of SIM2s and may be one mechanism for preventing progression of DCIS to invasive breast cancer[23].

\section{Discussion:}

Through transgenic mouse models and in vitro studies SIM2s has been identified as a novel player in several key aspects of mammary gland development. Specifically, genetic ablation of SIM2s in mammary epithelial cells revealed that SIM2s is required for ductal morphogenesis and differentiation of luminal cells for milk production during lactation. Furthermore, mammary specific overexpression of SIM2s resulted in a delay in post lactational mammary gland involution through suppression of Stat3 and NFKB signaling, as well as maintenance of a markers of epithelial cell differentiation normally observed only during lactation. These results suggest that SIM2s has tumor suppressive activities in the mammary gland through maintenance of epithelial cell differentiation. Consistent with this, loss of SIM2s expression in the mammary epithelium results in epithelial to mesenchymal transition (EMT) events, such as loss of E-cadherin and increases in matrix metalloprotease activity; results which are also observed in breast cancer cell lines. SIM2s is also downregulated in breast cancer patient samples further validating its potential role in tumor suppression[42]. In this study, we demonstrate a novel role for SIM2s as a negative regulator of the NFkB pathway, which normally results in downstream transcriptional activation of COX-2 expression. We have also shown that the SIM2s is targeted for suppression by NFKB signaling suggesting a regulatory feedback loop. Of particular interest, loss of SIM2s drastically increases pro-tumorigenic COX-2 expression and loss of COX-2 activity and expression results in re-expression of SIM2s. Thus, we have identified a reciprocal relationship between a molecule with known tumor suppressive activities, SIM2s, and known tumor promotional molecules NFkB and COX-2(Figure 5).

We predict that loss of SIM2s may be important for progression of in situ lesions to invasive disease. In a model of DCIS, loss of SIM2s is associated with an increase in invasiveness, enhanced tumor aggressiveness and progression. Specifically, upon loss of SIM2 in tumors an increase in colocalization of keratin 5 and vimentin have been observed[42], which is indicative of mesenchymal and invasive phenotypes. Additionally, matrix metalloproteinases (MMPs), which are associated with basement membrane degradation during mammary gland development and cancer, are also significantly increased with loss of SIM2[49-51]. Furthermore, P21, an important cell cycle regulator involved in both the p53 stress-response pathway and senescence, is markedly decreased mRNA in SIM2 knockdown xenografts[52, 53]. These changes likely promote an increased potential for progression of DCIS to IDC. Furthermore, increased COX-2 coupled with increased $\mathrm{p} 16$ and increased proliferation are associated subsequent recurrence of DCIS[22]. Here we show that cells with low invasive potential, the DCIS.COM and MCF7 cells, exhibit increased expression of COX-2 upon knockdown of SIM2s and endogenously express moderate levels of SIM2 compared to the low level of SIM2 observed in the more invasive SUM159 cells[38]. Likewise, overexpression of SIM2s in SUM159 cells decreases COX-2 expression. Coincidently, in a DCIS.COM xenograft study, SIM2s overexpression also significantly decreased COX-2 staining in tumor sections and xenografts from MCF10DCIS shCOX-2 cells exhibit increased expression of SIM2. These data demonstrate, for the first-time, a link between SIM2s expression and COX-2 and provide evidence of negative feedback of COX-2 on SIM2 expression. Since it is well known in the literature that COX-2 inhibition is associated with better prognosis for breast cancer patients[54,55], further studies on strategies for re-expression of SIM2s may be beneficial in improving prognosis of breast cancer patients. Furthermore, an additional implication is that SIM2s could be utilized as a 
marker to identify DCIS patients that are of low risk for acquisition of COX-2 expression and progression to IDC.

\section{Conclusions:}

These findings support a role for SIM2s in the prevention of breast cancer progression through its ability to interact with PTGS2 expression via modulating the NFKB signaling pathway. It has long been established that NFKB regulates genes involved in cell proliferation and cell survival. Specifically, blocking NFKB in tumor cells can lead to susceptibility from anti-cancer agents. However, due to the complexity of the tumor micro environment, NFKB signaling also has been found to have anti-cancer effects in various cancer cells. Thus, it is important to investigate a mechanism, specifically in mammary tissue, in which the targeted pathways are highly involved with cell proliferation, survival, migration and invasion. Due to elevated COX-2 expression correlating with poor prognosis, it is imperative to investigate reducing COX-2/PTGS2 expression. In the data provided here, we have demonstrated an integral role for SIM2s involvement in mediating NFKB signaling to decrease expression of COX-2/PTGS2 leading to an improved prognosis for breast cancer patients.

\section{Abbreviations:}

CHIP: Chromatin immunoprecipitation

COX-2: Cyclooxygenase 2

DCIS: Ductal carcinoma in situ

DHA: Docosahexaenoic acid

EMT: Epithelial mesenchymal transition

IDC: Invasive ductal carcinoma

IKB: Inhibitor kappa b

IKB $\alpha$ : Inhibitor kappa b alpha

IKB-SR: Inhibitor kappa b alpha super repressor

IKK $\alpha$ : Inhibitor kappa b kinase alpha

IKK $\beta$ : Inhibitor kappa b kinase beta

NFkB: Nuclear factor kappa b

PUFA: polyunsaturated fatty acids

SIM2s: Singleminded-2s

\section{Declarations:}

\section{Acknowledgements}

We gratefully acknowledge Kornelia Polyak and Andriy Marusyk for providing the shCOX-2 DCIS cell lines

\section{Funding}

This research is supported by an NRSA F31CA236140 (LC/TL), the University of Colorado Department of Medicine Outstanding Early Career Scholars Program (TL) and the National Cancer Institute R21CA197896 (WP); R01HD083952 (CO-PI WP, MR), R21CA185226 (TL), and R01CA211696 (TL).

\section{Availability of data and materials}

Not applicable

\section{Authors' contributions}

GW performed the in vitro experiments, formal data analysis, interpretation of the data, production of figures, writing of the original draft, as well as reviewing and editing the final draft of the manuscript.CY and LC were involved in tumor immunostaining and immunoblot analysis. VW was involved in designing and performing immunostaining of the xenograft studies. TG was involved in animal husbandry, in vivo experiments and CHiP analysis. YF and RC provided the polyunsaturated fatty acid resources. WP and TL oversaw all the experiments and experimental design, interpretation of the data, provided the resources, and contributed to the writing as well as reviewing and editing of the manuscript. All authors read and approved the final manuscript. 


\section{Ethics approval and consent to participate}

Animal studies were approved by the Institutions laboratory care committee.

\section{Consent for publication}

Not applicable

\section{Competing Interests}

The authors declare that they have no competing interests.

1. Siegel, R.L., K.D. Miller, and A. Jemal, Cancer statistics, 2018. CA Cancer J Clin, 2018. 68(1): p. 7-30.

2. Cowell, C.F., et al., Progression from ductal carcinoma in situ to invasive breast cancer: revisited. Mol Oncol, 2013. 7(5): p. 859-69.

3. Schedin, P., et al., Microenvironment of the involuting mammary gland mediates mammary cancer progression. J Mammary Gland Biol Neoplasia, 2007. 12(1): p. 71-82.

4. Pensa, S., C.J. Watson, and V. Poli, Stat3 and the inflammation/acute phase response in involution and breast cancer. J Mammary Gland Biol Neoplasia, 2009. 14(2): p. 121-9.

5. Clarkson, R.W. and C.J. Watson, Microarray analysis of the involution switch. J Mammary Gland Biol Neoplasia, 2003. 8(3): p. 309-19.

6. Ernster, V.L., et al., Detection of ductal carcinoma in situ in women undergoing screening mammography. J Natl Cancer Inst, 2002. 94(20): p. 1546-54.

7. ACS. American Cancer Society: Cancer Facts and Figures 2013. American Cancer Society 2013 January 6 , 2012; Available from: http://www.cancer.org/research/cancerfactsfigures/cancerfactsfigures/cancerfacts-figures-2012.

8. Narod, S.A., et al., Breast Cancer Mortality After a Diagnosis of Ductal Carcinoma In Situ. JAMA Oncol, 2015.

9. Kerlikowske, K., et al., Characteristics associated with recurrence among women with ductal carcinoma in situ treated by lumpectomy. J Natl Cancer Inst, 2003. 95(22): p. 1692-702.

10. Esserman, L. and C. Yau, Rethinking the Standard for Ductal Carcinoma In Situ Treatment. JAMA Oncol, 2015.

11. Hanahan, D. and R.A. Weinberg, Hallmarks of cancer: the next generation. Cell, 2011. 144(5): p. 646-74.

12. Fitzpatrick, S.F., et al., An intact canonical NF-kappaB pathway is required for inflammatory gene expression in response to hypoxia. J Immunol, 2011. 186(2): p. 1091-6.

13. Schmedtje, J.F., Jr., et al., Hypoxia induces cyclooxygenase-2 via the NF-kappaB p65 transcription factor in human vascular endothelial cells. J Biol Chem, 1997. 272(1): p. 601-8.

14. Newton, R., et al., Evidence for involvement of NF-kappaB in the transcriptional control of COX-2 gene expression by IL-1beta. Biochem Biophys Res Commun, 1997. 237(1): p. 28-32.

15. Nguyen, L.K., et al., Species differential regulation of COX2 can be described by an NFkappaBdependent logic AND gate. Cell Mol Life Sci, 2015. 72(12): p. 2431-43.

16. Hayden, M.S. and S. Ghosh, Shared principles in NF-kappaB signaling. Cell, 2008. 132(3): p. 344-62.

17. Hugo, H.J., et al., New Insights on COX-2 in Chronic Inflammation Driving Breast Cancer Growth and Metastasis. Journal of Mammary Gland Biology and Neoplasia, 2015. 20(3): p. 109-119.

18. Griswold, D.E. and J.L. Adams, Constitutive cyclooxygenase (COX-1) and inducible cyclooxygenase (COX2): rationale for selective inhibition and progress to date. Med Res Rev, 1996. 16(2): p. 181-206.

19. Ristimaki, A., et al., Prognostic significance of elevated cyclooxygenase-2 expression in breast cancer. Cancer Res, 2002. 62(3): p. 632-5.

20. Denkert, C., et al., Elevated expression of cyclooxygenase-2 is a negative prognostic factor for disease free survival and overall survival in patients with breast carcinoma. Cancer, 2003. 97(12): p. 2978-87. 
21. Spizzo, G., et al., Correlation of COX-2 and Ep-CAM overexpression in human invasive breast cancer and its impact on survival. Br J Cancer, 2003. 88(4): p. 574-8.

22. Gauthier, M.L., et al., Abrogated response to cellular stress identifies DCIS associated with subsequent tumor events and defines basal-like breast tumors. Cancer Cell, 2007. 12(5): p. 479-91.

23. Lyons, T.R., et al., Postpartum mammary gland involution drives progression of ductal carcinoma in situ through collagen and COX-2. Nat Med, 2011. 17(9): p. 1109-15.

24. Lyons, T.R., et al., Cyclooxygenase-2-dependent lymphangiogenesis promotes nodal metastasis of postpartum breast cancer. J Clin Invest, 2014. 124(9): p. 3901-12.

25. Black, S.A., et al., Semaphorin 7a exerts pleiotropic effects to promote breast tumor progression. Oncogene, 2016. 35(39): p. 5170-8.

26. Costa, C., et al., Cyclo-oxygenase 2 expression is associated with angiogenesis and lymph node metastasis in human breast cancer. J Clin Pathol, 2002. 55(6): p. 429-34.

27. Esbona, K., et al., COX-2 modulates mammary tumor progression in response to collagen density. Breast Cancer Research, 2016. 18(1): p. 35.

28. Harris, R.E., B.C. Casto, and Z.M. Harris, Cyclooxygenase-2 and the inflammogenesis of breast cancer. World J Clin Oncol, 2014. 5(4): p. 677-92.

29. Mann, J.R., M.G. Backlund, and R.N. DuBois, Mechanisms of disease: Inflammatory mediators and cancer prevention. Nat Clin Pract Oncol, 2005. 2(4): p. 202-10.

30. Sheehan, K.M., et al., The relationship between cyclooxygenase-2 expression and colorectal cancer. Jama, 1999. 282(13): p. 1254-7.

31. Visscher, D.W., et al., Association between cyclooxygenase-2 expression in atypical hyperplasia and risk of breast cancer. J Natl Cancer Inst, 2008. 100(6): p. 421-7.

32. Bourn, J. and M. Cekanova, Cyclooxygenase inhibitors potentiate receptor tyrosine kinase therapies in bladder cancer cells in vitro. Drug Des Devel Ther, 2018. 12: p. 1727-1742.

33. Buttar, N.S., et al., Chemoprevention of esophageal adenocarcinoma by COX-2 inhibitors in an animal model of Barrett's esophagus. Gastroenterology, 2002. 122(4): p. 1101-12.

34. Wang, W. and J. Wang, Toll-Like Receptor 4 (TLR4)/Cyclooxygenase-2 (COX-2) Regulates Prostate Cancer Cell Proliferation, Migration, and Invasion by NF-kappaB Activation. Med Sci Monit, 2018. 24: p. 5588-5597.

35. Wu, C., et al., IL-1beta-Mediated Up-Regulation of WT1D via miR-144-3p and Their Synergistic Effect with NF-kappaB/COX-2/HIF-1alpha Pathway on Cell Proliferation in LUAD. Cell Physiol Biochem, 2018. 48(6): p. 2493-2502.

36. Zeng, X. and S. Yi, Cyclooxygenase Inhibitors in Epithelial Ovarian Cancer Treatment. Int J Gynecol Cancer, 2018. 28(6): p. 1085-1089.

37. Gustafson, T.L., et al., Ha-Ras transformation of MCF10A cells leads to repression of Singleminded-2s through NOTCH and C/EBPbeta. Oncogene, 2009.

38. Kwak, H.I., et al., Inhibition of breast cancer growth and invasion by single-minded 2s. Carcinogenesis, 2007. 28(2): p. 259-66.

39. Laffin, B., et al., Loss of singleminded-2s in the mouse mammary gland induces an epithelialmesenchymal transition associated with up-regulation of slug and matrix metalloprotease 2. Mol Cell Biol, 2008. 28(6): p. 1936-46.

40. Scribner, K.C., F. Behbod, and W.W. Porter, Regulation of DCIS to invasive breast cancer progression by Singleminded-2s (SIM2s). Oncogene, 2012.

41. Scribner, K.C., et al., Singleminded-2s (Sim2s) promotes delayed involution of the mouse mammary gland through suppression of Stat3 and NFkappaB. Mol Endocrinol, 2011. 25(4): p. 635-44.

42. Scribner, K.C., F. Behbod, and W.W. Porter, Regulation of DCIS to invasive breast cancer progression by Singleminded-2s (SIM2s). Oncogene, 2013. 32(21): p. 2631-9. 
43. Miller, F.R., et al., MCF10DCIS.com xenograft model of human comedo ductal carcinoma in situ. J Natl Cancer Inst, 2000. 92(14): p. 1185-6.

44. Bouwens, M., et al., Fish-oil supplementation induces antiinflammatory gene expression profiles in human blood mononuclear cells. Am J Clin Nutr, 2009. 90(2): p. 415-24.

45. Calviello, G., et al., n-3 PUFAs reduce VEGF expression in human colon cancer cells modulating the COX2/PGE2 induced ERK-1 and -2 and HIF-1alpha induction pathway. Carcinogenesis, 2004. 25(12): p. 2303-10.

46. Lee, S.A., et al., DHA and EPA Down-regulate COX-2 Expression through Suppression of NF-kappaB Activity in LPS-treated Human Umbilical Vein Endothelial Cells. Korean J Physiol Pharmacol, 2009. 13(4): p. 301-7.

47. Al-Jawadi, A., et al., Protective properties of $n-3$ fatty acids and implications in obesity-associated breast cancer. J Nutr Biochem, 2018. 53: p. 1-8.

48. Straka, S., et al., Incorporation of eicosapentaenioic and docosahexaenoic acids into breast adipose tissue of women at high risk of breast cancer: a randomized clinical trial of dietary fish and n-3 fatty acid capsules. Mol Nutr Food Res, 2015. 59(9): p. 1780-90.

49. Liu, D., et al., Association between polymorphisms in the promoter regions of matrix metalloproteinases (MMPs) and risk of cancer metastasis: a meta-analysis. PLoS One, 2012. 7(2): p. e31251.

50. Mendes, O., H.T. Kim, and G. Stoica, Expression of MMP2, MMP9 and MMP3 in breast cancer brain metastasis in a rat model. Clin Exp Metastasis, 2005. 22(3): p. 237-46.

51. Barajas-Castaneda, L.M., et al., Overexpression of MMP-3 and UPA with Diminished PAI-1 Related to Metastasis in Ductal Breast Cancer Patients Attending a Public Hospital in Mexico City. J Immunol Res, 2016. 2016: p. 8519648.

52. Giordano, C., et al., Benzofuran-2-acetic ester derivatives induce apoptosis in breast cancer cells by upregulating p21(Cip/WAF1) gene expression in p53-independent manner. DNA Repair (Amst), 2017. 51: p. 20-30.

53. Mauro, M., et al., p21 promotes error-free replication-coupled DNA double-strand break repair. Nucleic Acids Res, 2012. 40(17): p. 8348-60.

54. Kwan, M.L., et al., NSAIDs and breast cancer recurrence in a prospective cohort study. Cancer Causes Control, 2007. 18(6): p. 613-20.

55. Li, J., et al., Celecoxib in breast cancer prevention and therapy. Cancer Manag Res, 2018. 10: p. 46534667. 
Figure 1. A. Luciferase activity in HEK293T cells co-transfected with $5 x \mathrm{kB}$ binding sites upstream of the luciferase gene (5x NFkB-luc) and NFkB p65 and/or SIM2s. (Diagram of promoter construct is shown above for reference.) B. Luciferase activity in HEK293T cells co-transfected with 5x NFkB-luc and NFkB p65 and/or SIM2s with its repression domain deleted (SIM2s $\triangle$ R). C. SUM159 plpcx emp (control), SUM159 plpcx SIM2s-FLAG (overexpression), MCF7 psil SCR (control), and MCF7 psil SIM2-shRNA (knock down) were analyzed by immunoblot for levels of IKKA, IKKB, phospho-p65, p65 and Beta Actin as loading control. Unpaired $t$ test: ${ }^{*}, P<$ $0.02 ;{ }^{* *}, P<0.05$. Analysis performed via ImageJ for comparison of protein expression.

Figure 2. A. SIM2s expression in DCIS.COM control cells and cells overexpressing IKKB by qPCR as fold change. B. SIM2s expression in DCIS.COM control cells and cells overexpressing IKB-SR by qPCR as fold change. C. SIM2 promoter activity in HEK293T cells co-transfected with SIM2 promoter upstream of the luciferase gene and increasing amounts of NFKB p65 (50uM,100uM, and 150uM). D. SIM2 promoter activity after co-transfection of promoter with control vector (pcDNA3), NFkB p65, and/or IkB-SR. E. ChIP assay for NFkB p65 binding after transient transfection of SIM2 promoter with NFKB p65 in HEK293T cells. F. SIM2 promoter activity in HEK293T Cells co-transfected with SIM2 promoter upstream of the luciferase gene and 150uM NFKB p65 compared with the SIM2 promoter activity in HEK293T cells co-transfected with NFKB double mutant SIM2 promoter upstream of the luciferase gene. Unpaired $t$ test: ${ }^{*}, P<0.05$.

Figure 3. A. PTGS2 expression in MCF7 control cells and cells overexpressing SIM2s by real time qPCR as fold change. B. PTGS2 expression in DCIS.COM control cells and cells with SIM2-shRNA by real time qPCR as fold change. C. PTGS2 expression in SUM159 control cells and cells overexpressing SIM2s by real time qPCR as fold change. D. Immunohistochemistry for COX-2 in DCIS.COM cells stably transduced with control vector, SIM2s-FLAG (overexpression), respectively. Unpaired $t$ test: ${ }^{*}, P<0.01$.

Figure 4. A. DCIS.COM control, 1 shPTGS2, 2 shPTGS2 were analyzed by western blot for SIM2 and GAPDH as loading control. B. IHC Analysis for SIM2 positive nuclei in tumors generated from control and shPTGS2 DCIS.COM cells. C. Images of IHC analysis for SIM2 in Tumors generated from control and shPTGS2 DCIS.COM cells. D. SIM2s expression in SUM159 control cells and cells dosed with 20uM Celecoxib by qPCR as fold change. E. SIM2s expression in DCIS.COM control cells and cells dosed with 50uM DHA by qPCR as fold change. Unpaired $t$ test: ${ }^{*}, P<0.05$.

Figure 5. A model depicting NFkB-mediated SIM2 signaling. Our current data suggest that NFkB inhibits SIM2 expression through an unknown mechanism to result in upregulation of COX-2 expression and subsequent activity, which further downregulates SIM2. SIM2 is also capable of decreasing NFKB signaling to down regulate COX-2 expression.

\section{Supplemental Figure Legends}

SFigure 1. qRTPCR analysis of PTGS2 expression in 3 different breast cancer cell lines.

STable 1. Antibody list for immunoblot and immunohistochemical analyses.

Stable 2. Primer sequences for qPCR analyses. 
Figure 1

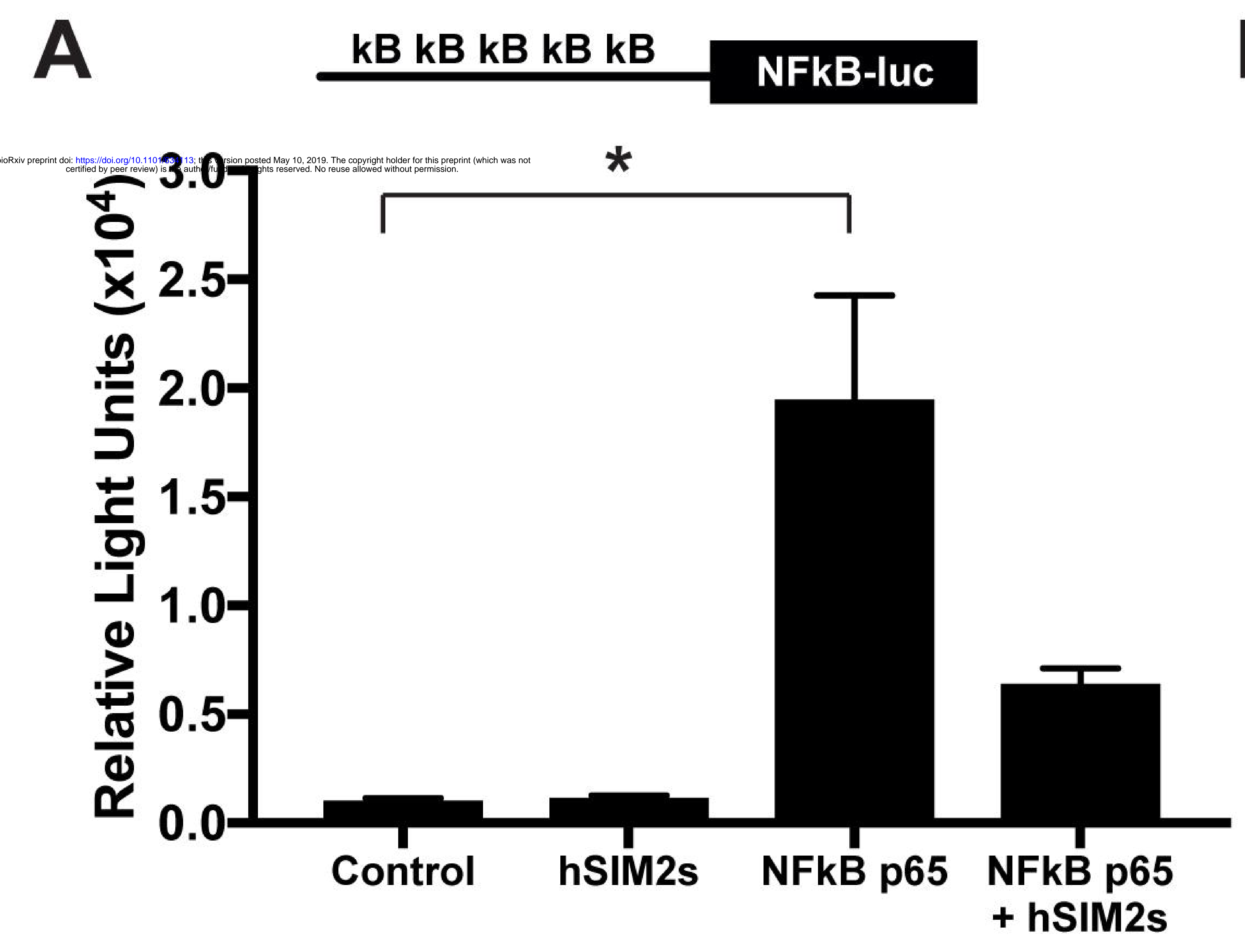

B kB kB kB kB kB

NFkB-luc

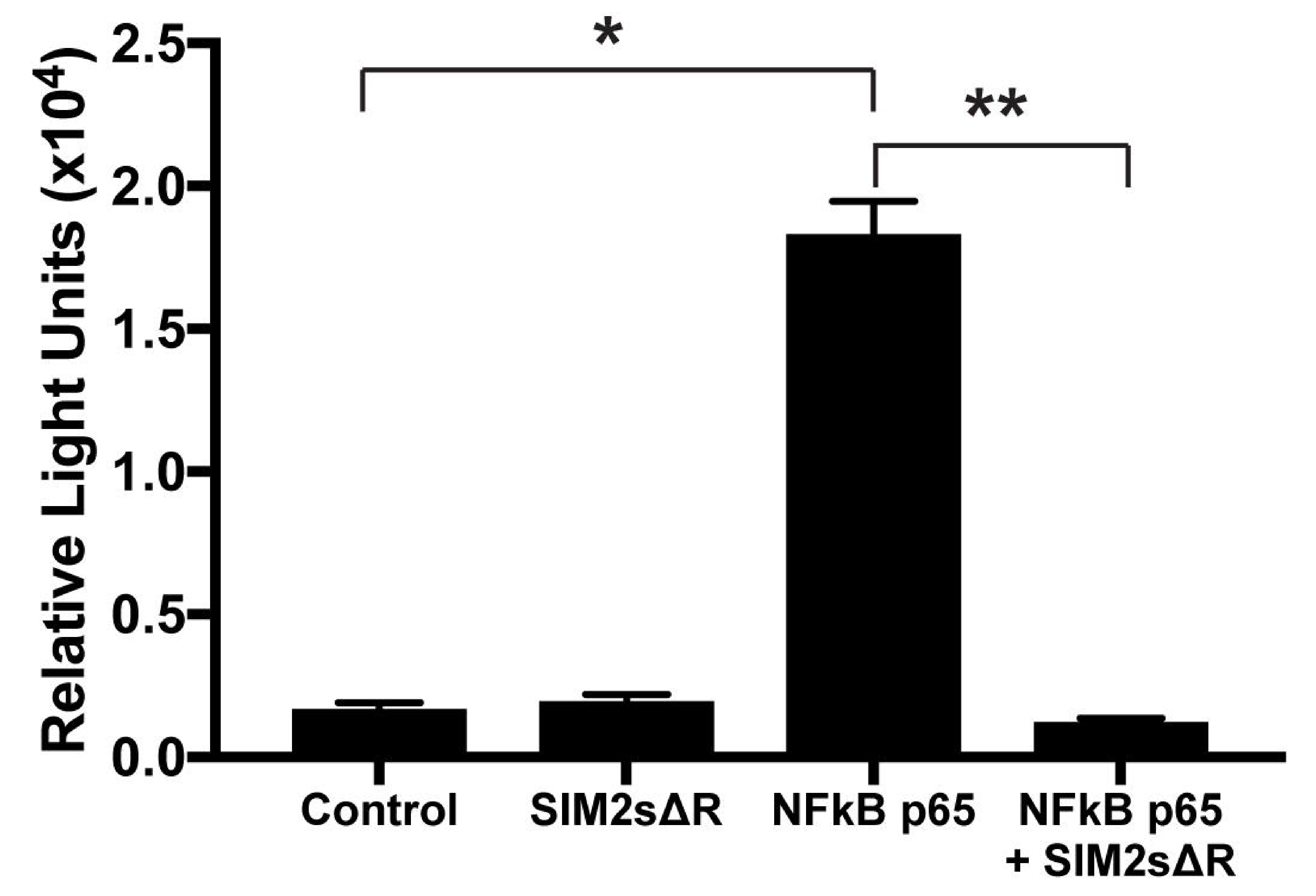

C

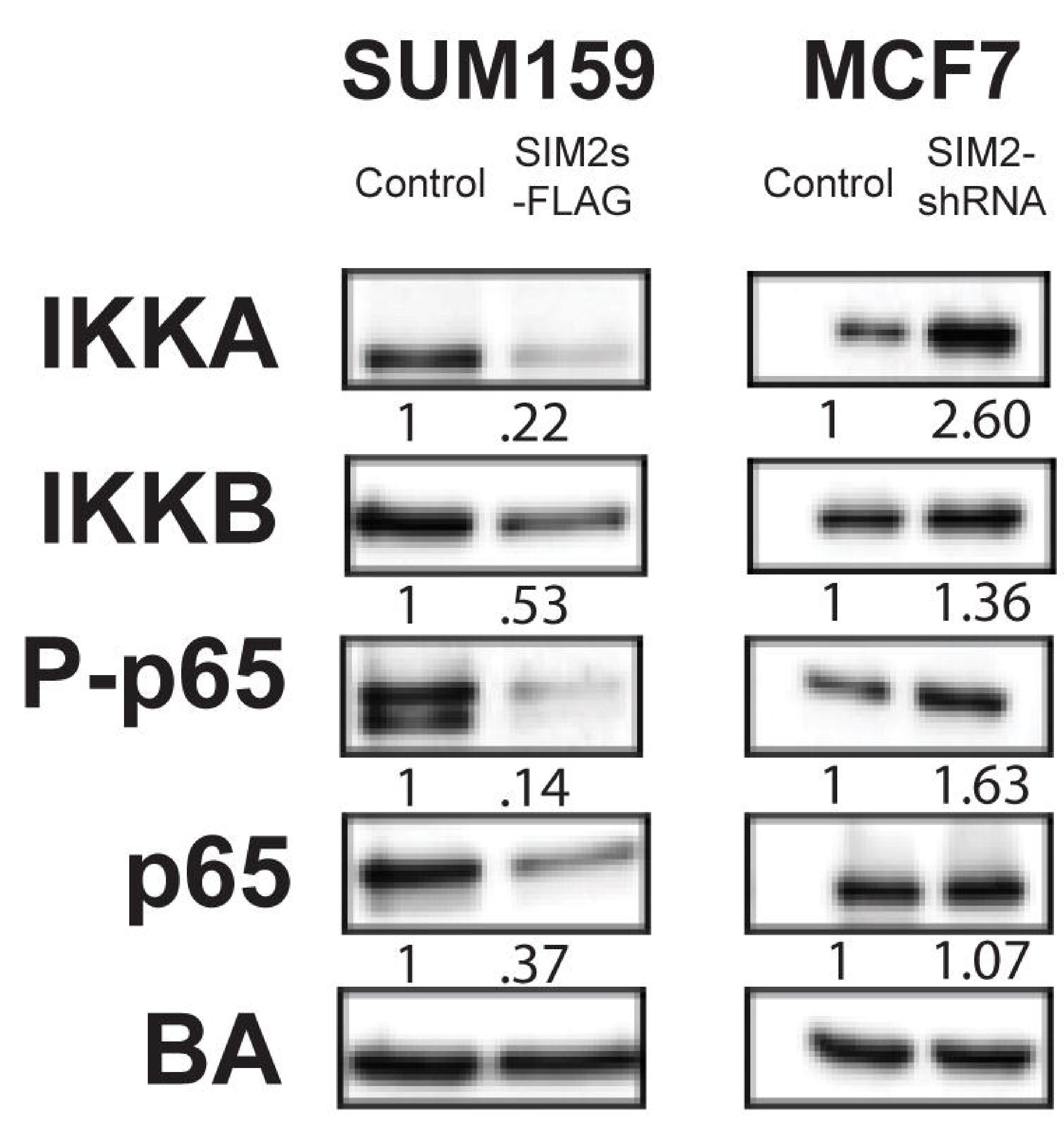


A

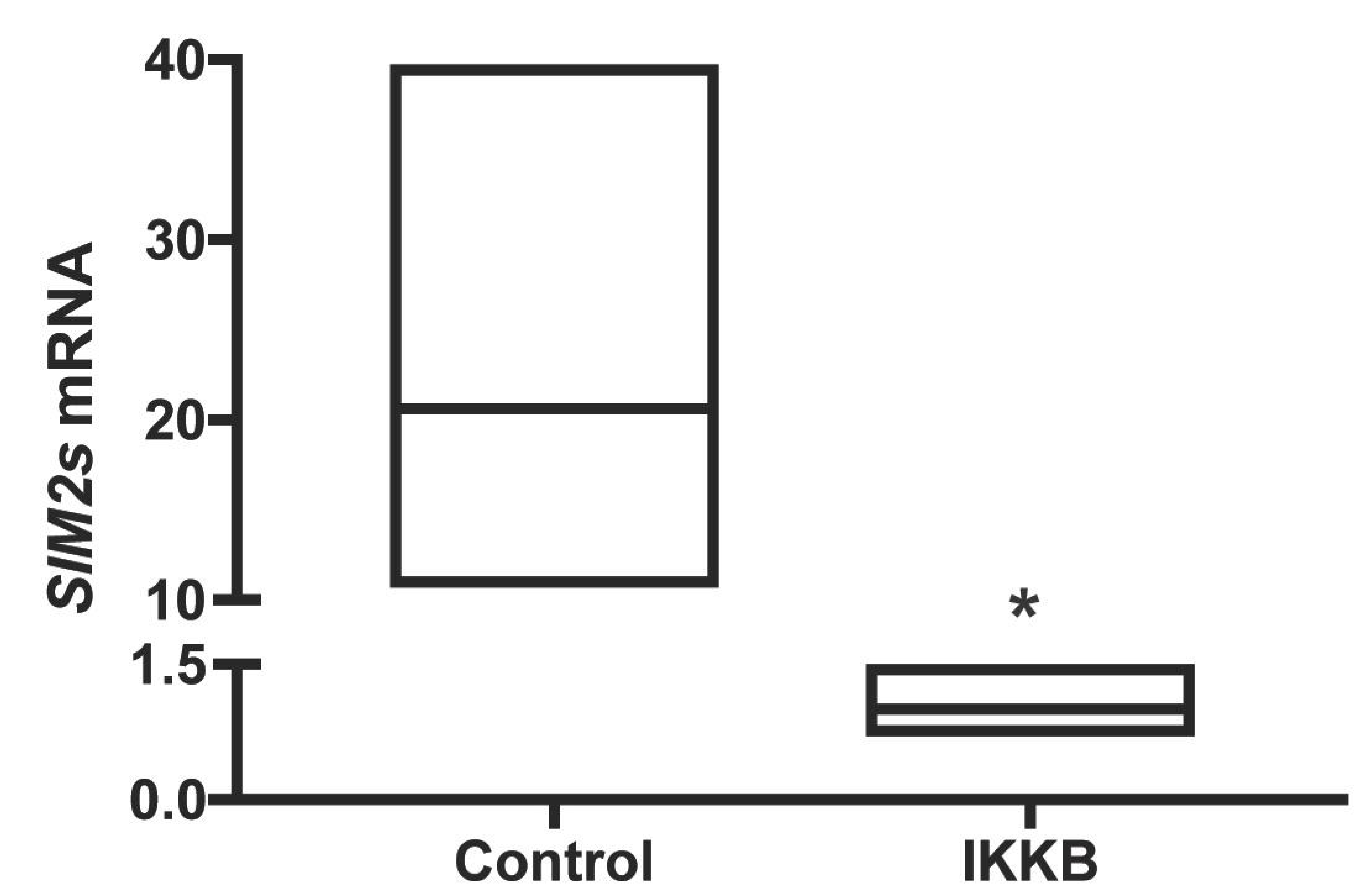

C
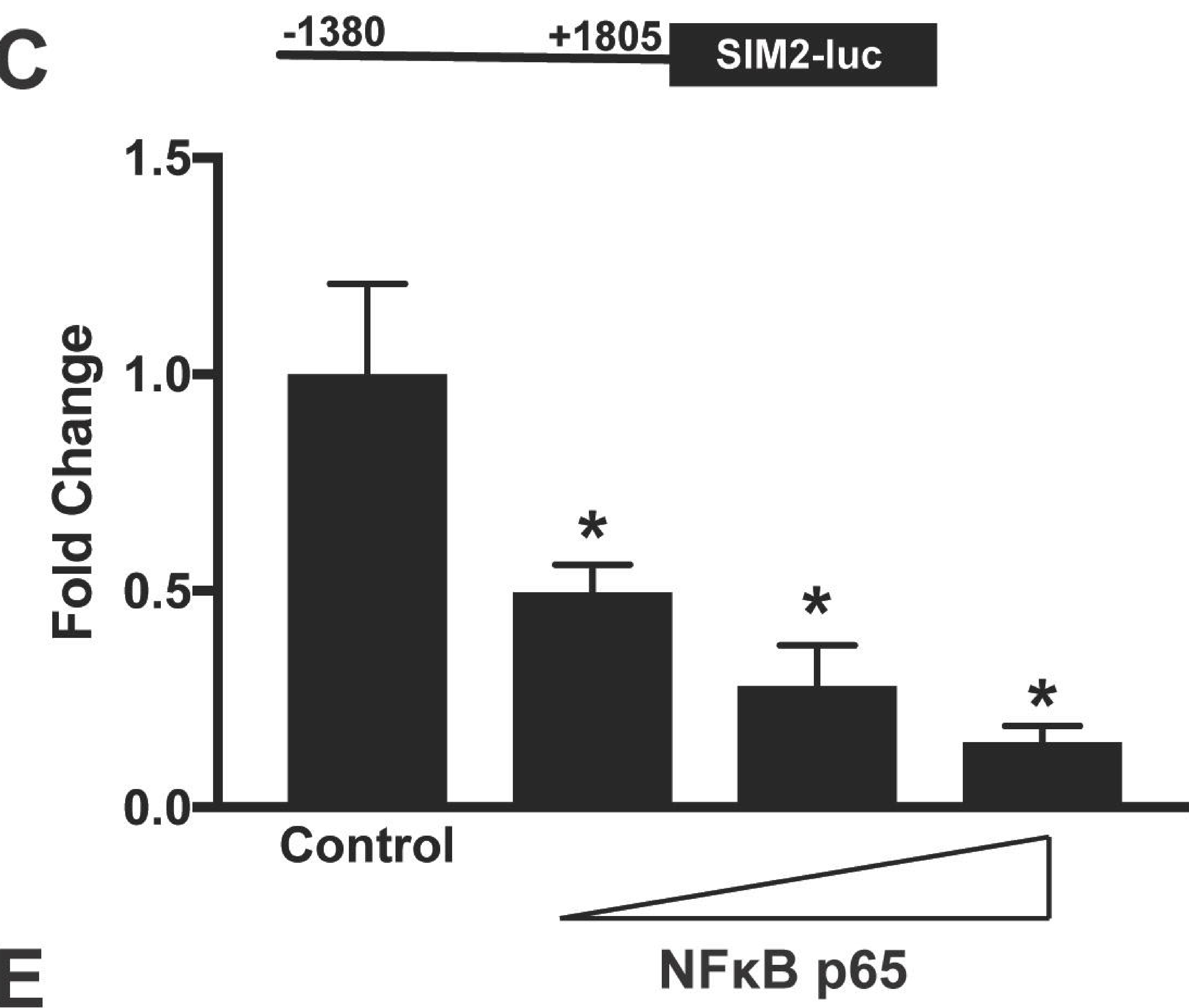

E

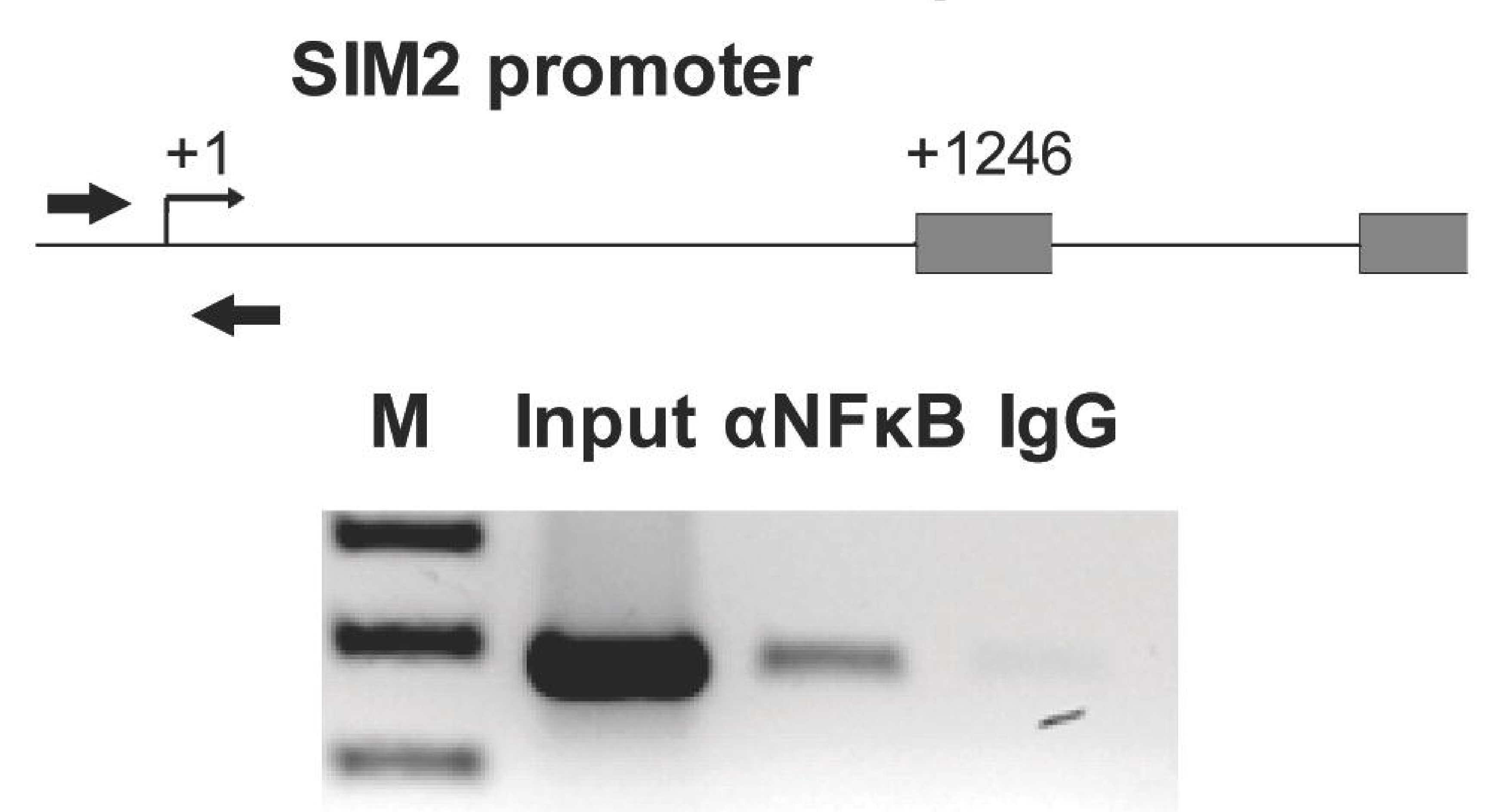

B
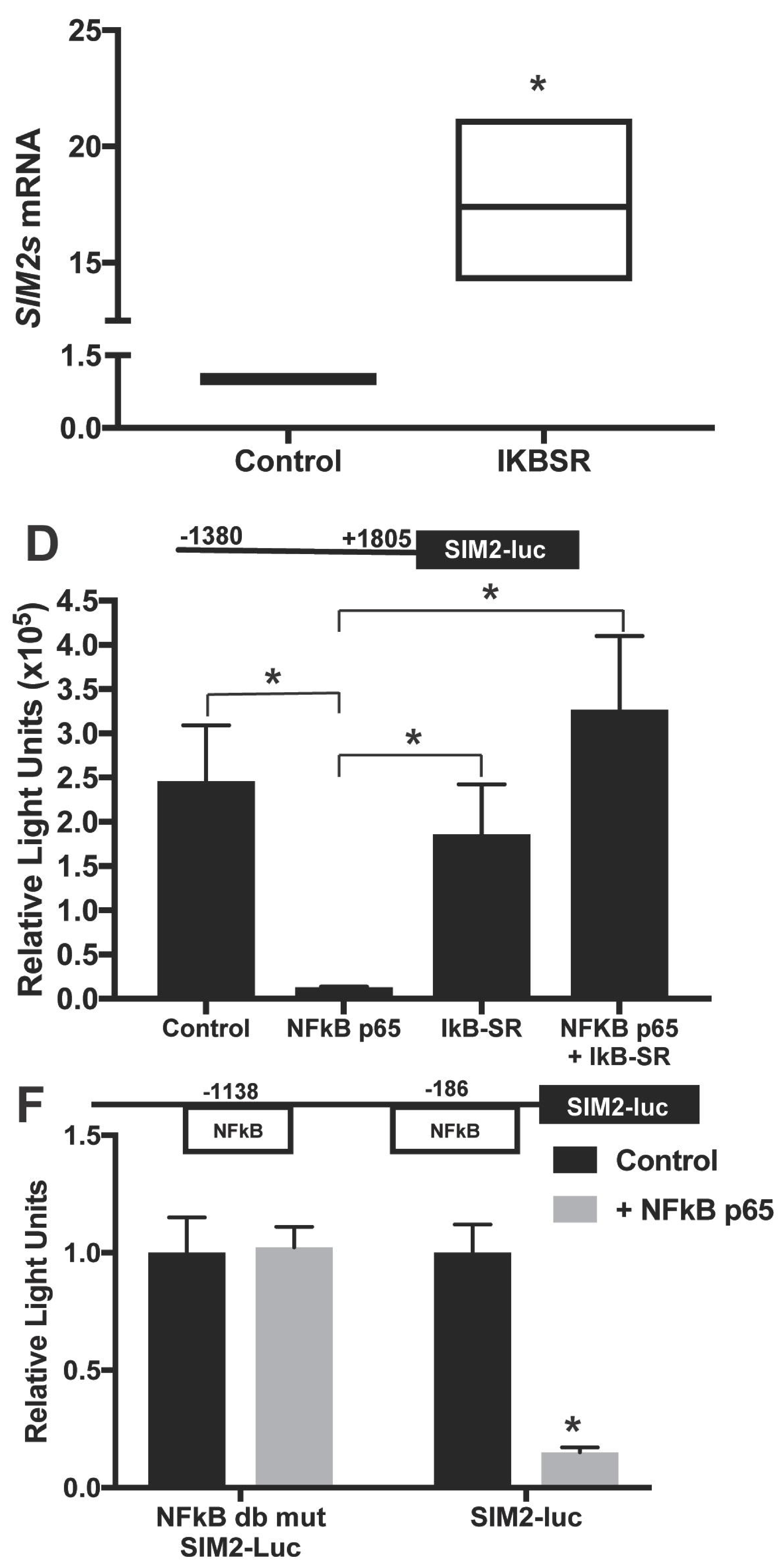
Figure 3
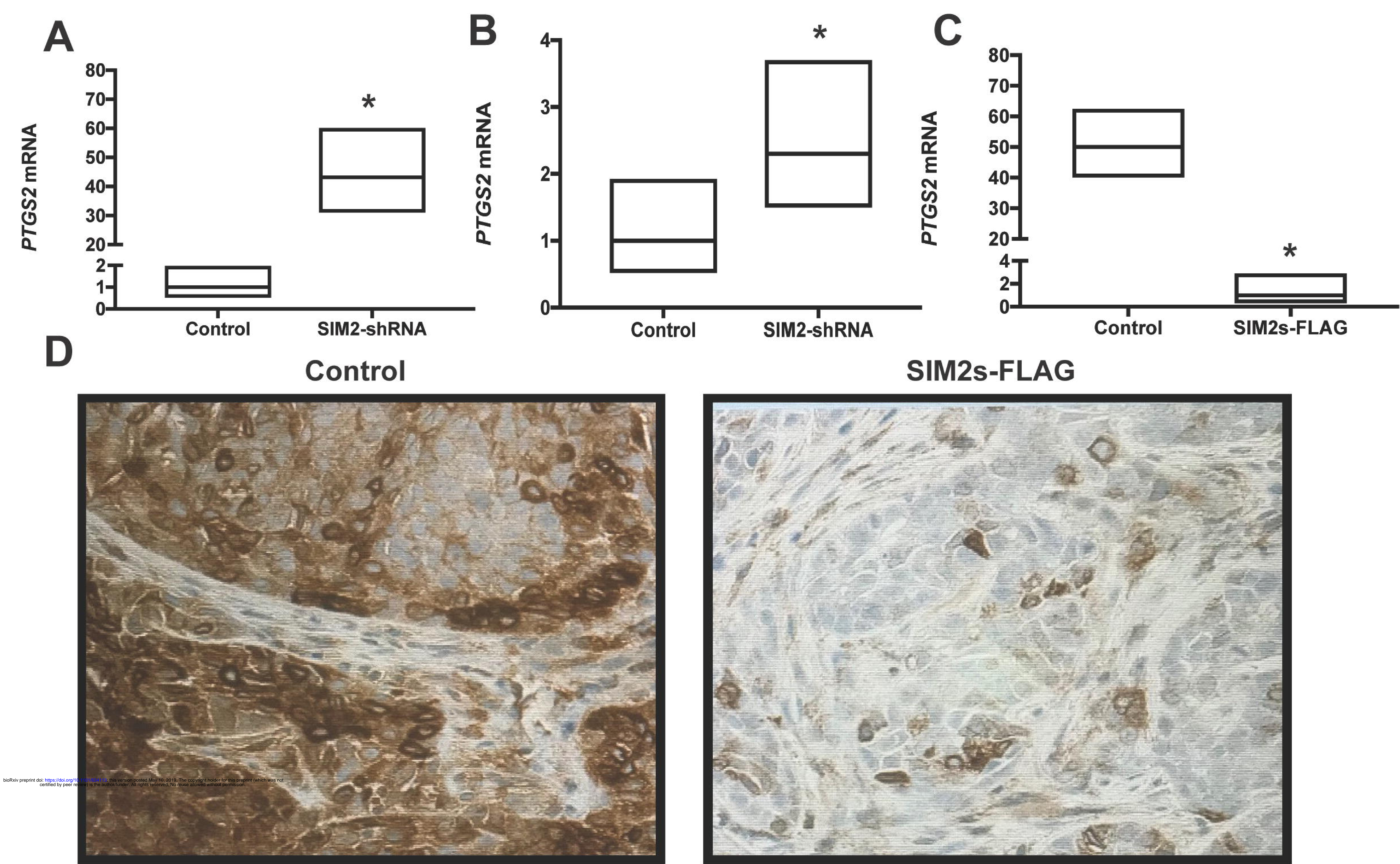


\section{A}

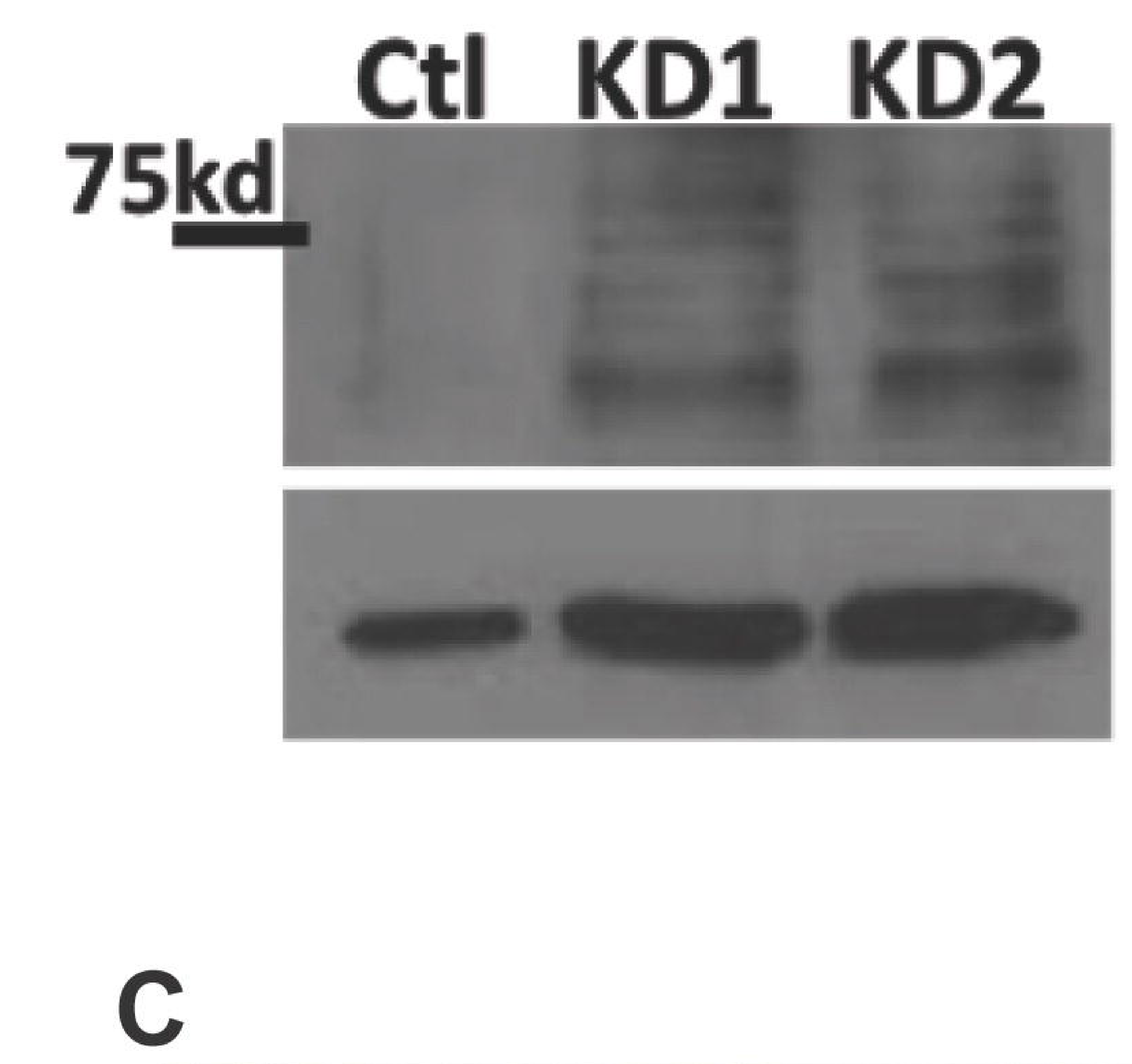

Control

D

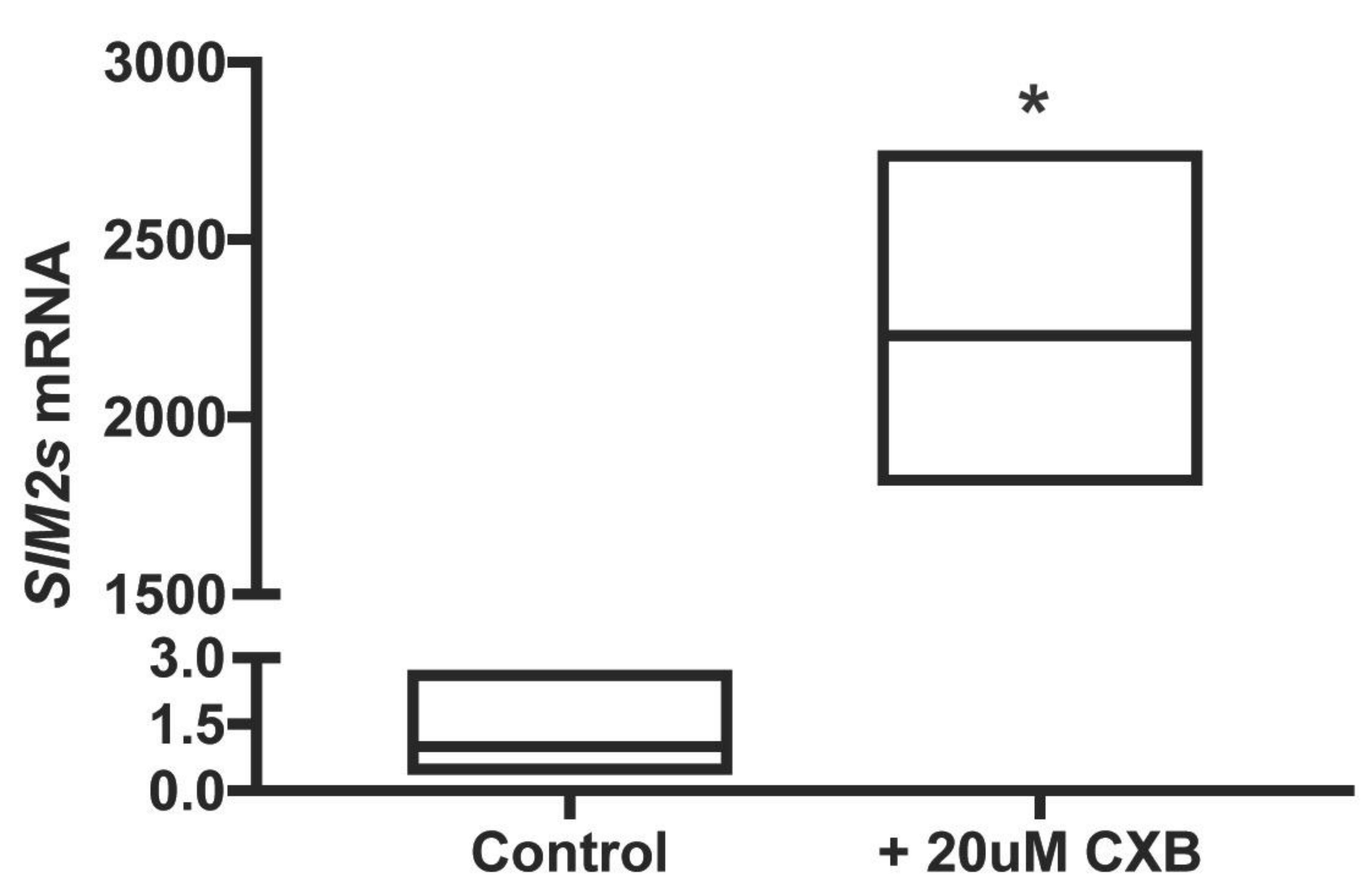

Sim-2

GAPDH
B
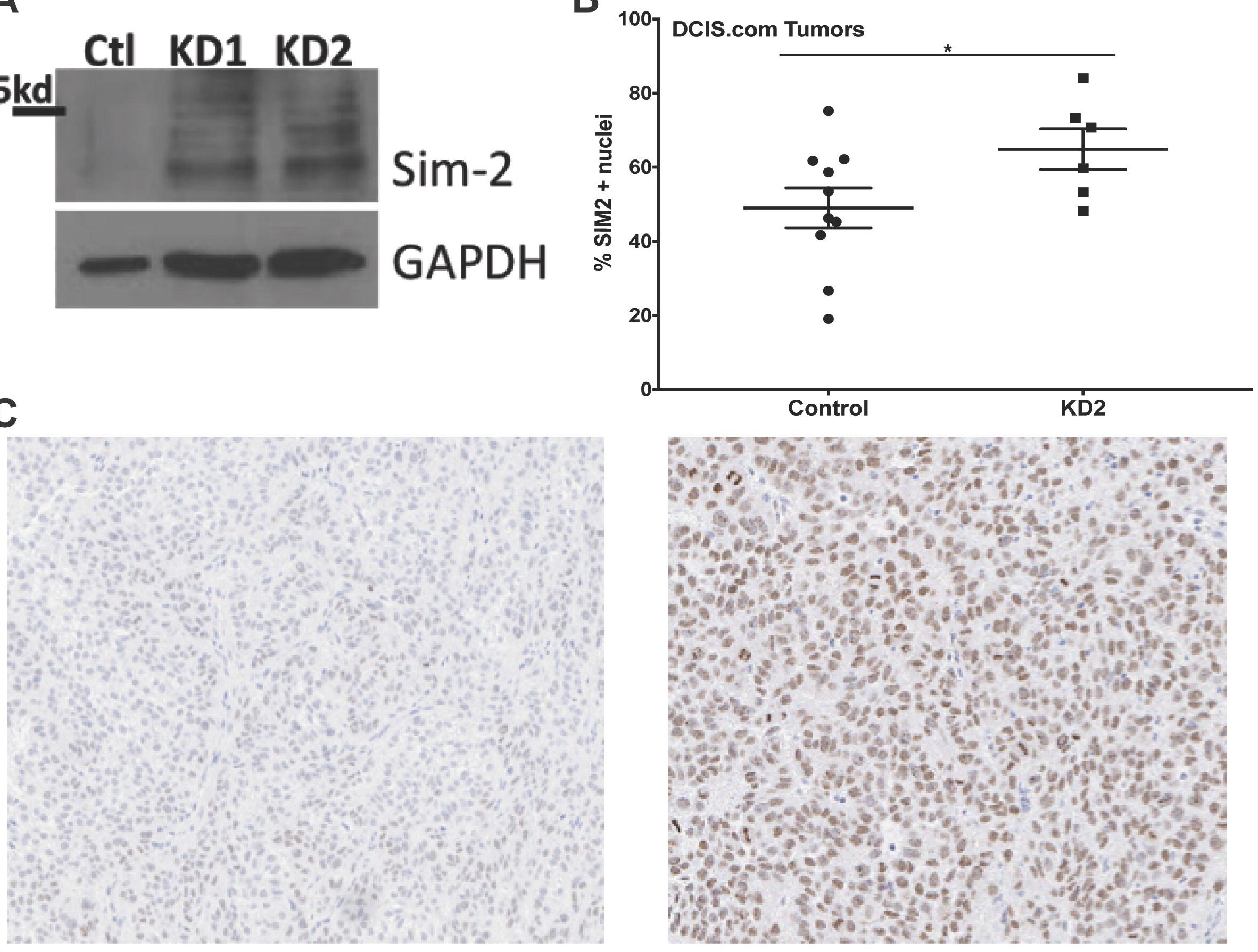

KD2

E

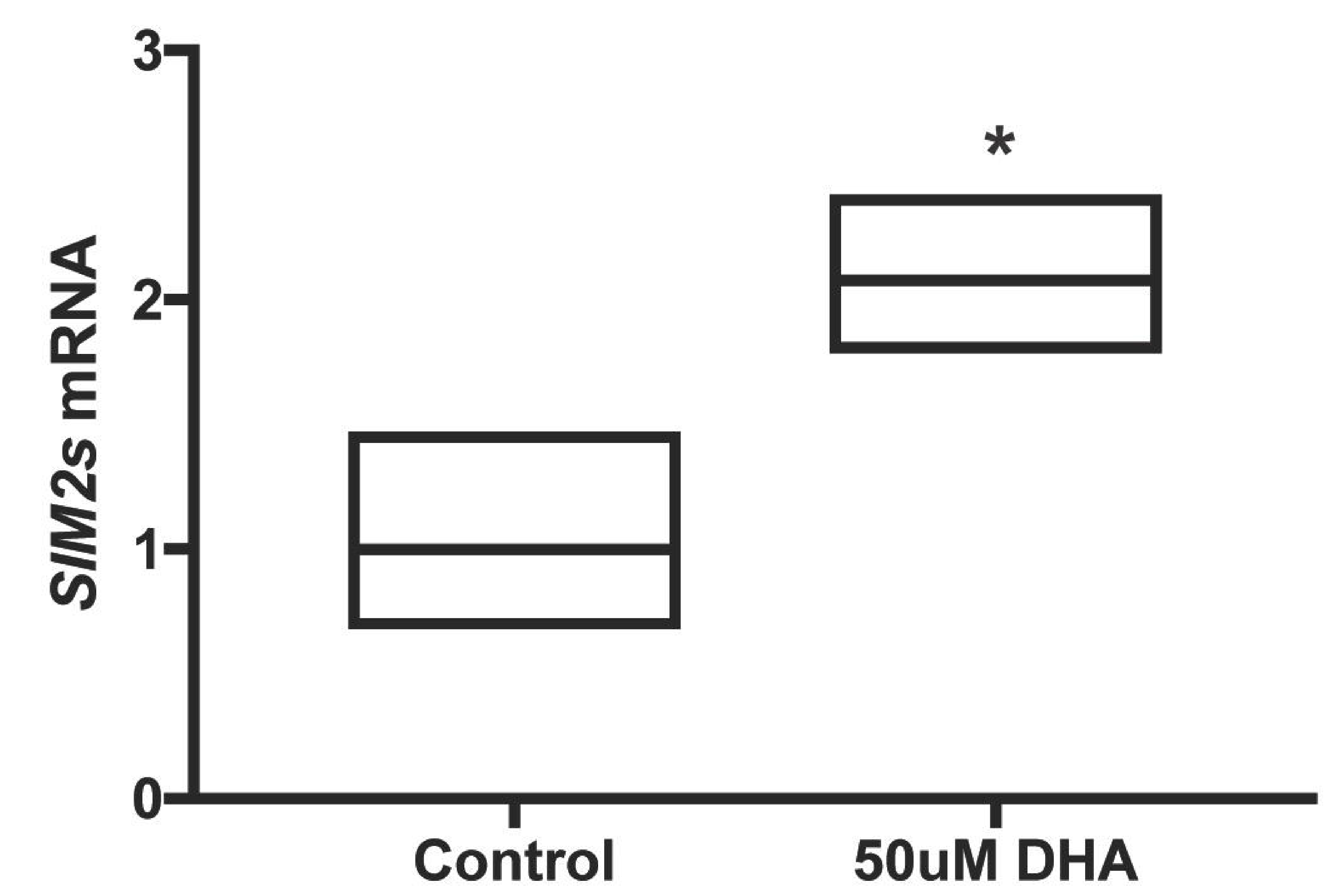


Nuclear Factor Kappa B
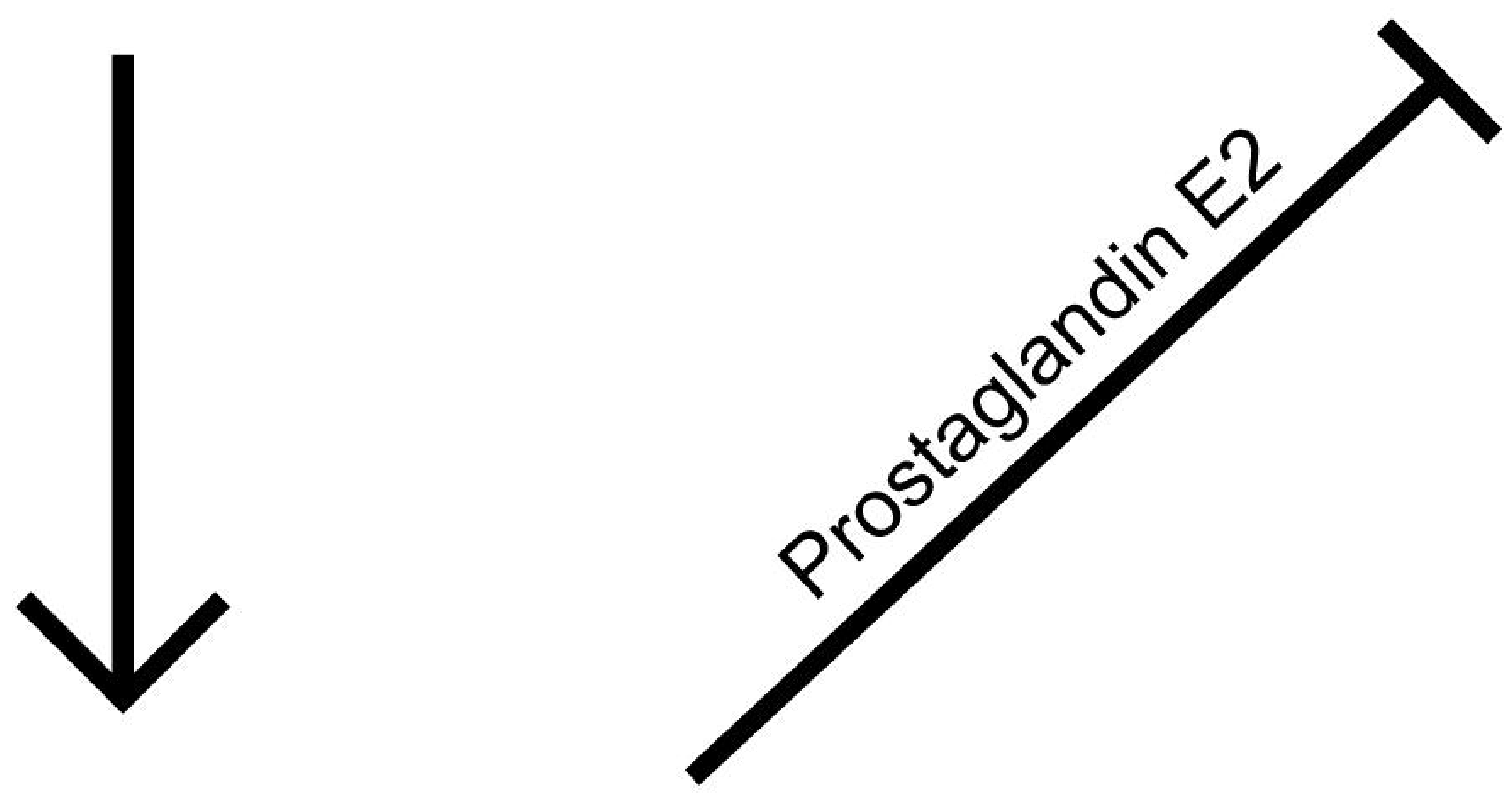

Singleminded 2s

Cyclooxygenase 2 